Proyecciones Journal of Mathematics

Vol. 36, No 2, pp. 245-255, June 2017.

Universidad Católica del Norte

Antofagasta - Chile

\title{
Fuzzy normed linear space valued sequence space $\ell_{p}^{F}(X) *$
}

\author{
Paritosh Chandra Das \\ Rangia College, India \\ Received : February 2016. Accepted : March 2017
}

\begin{abstract}
In this article we define the notion of fuzzy normed linear space valued sequence space $\ell_{p}^{F}(X), 1 \leq p<\infty$ in a fuzzy normed linear space $X$ and discuss some of its properties like completeness, monotone, solidness, convergence free and symmetricity. Also we prove some inclusion results.
\end{abstract}

Key Words : Fuzzy real number, fuzzy normed linear space, monotone, solid space, convergence free and symmetricity.

Mathematics Subject Classification (2010) : 40A05, 40D25, 03E72.

${ }^{*}$ The work of the author is supported by University Grants Commission of India vide project No. F. 42-28/2013(SR), dated- 12 MARCH, 2013. 


\section{Introduction}

The concept of fuzzy set, a set whose boundary is not sharp or precise has been introduced by L. A. Zadeh in 1965. It is the origin of new theory of uncertainty, distinct from the notion of probability. After the introduction of fuzzy sets, the scope for studies in different branches of pure and applied mathematics increased widely. The notion of fuzzy set theory has been applied to introduce the notion of fuzzy real numbers which helps in constructing the sequence of fuzzy real numbers. Different types of sequence spaces of fuzzy real numbers have been studied under classical metric by Das ([1], [2]), Matloka [5], Subrahmanyam [6], Tripathy et al [7], Tripathy and Dutta ([8], [9]), Tripathy and Sarma [10], Tripathy and Debnath [11] and many others. A few works on fuzzy norm which has relationship with fuzzy metric have been done by Felbin [3] and some others. There is a lot of material for the classes of sequences those can be examined by fuzzy norm.

\section{Definitions and preliminaries}

A fuzzy real number $X$ is a fuzzy set on $R$, i.e. a mapping $X: R \rightarrow I(=$ $[0,1])$ associating each real number $t$ with its grade of membership $X(t)$.

A fuzzy real number $X$ is called convex if $X(t) \geq X(s) \wedge X(r)=\min$ $(X(s), X(r))$, where $s<t<r$.

If there exists $t_{0} \in R$ such that $X\left(t_{0}\right)=1$, then the fuzzy real number $X$ is called normal.

A fuzzy real number $X$ is said to be upper-semi continuous if, for each $\varepsilon>0, X^{-1}([0, a+\varepsilon))$, for all $a \in I$ is open in the usual topology of $R$.

The set of all upper-semi continuous, normal, convex fuzzy real numbers is denoted by $R(I)$. Throughout the article, by a fuzzy real number we mean that the number belongs to $R(I)$.

The $\alpha$-level set $[X]^{\alpha}$ of the fuzzy real number $X$, for $0<\alpha \leq 1$, defined as $[X]^{\alpha}=\{t \in R: X(t) \geq \alpha\}$. If $\alpha=0$, then it is the closure of the strong 0 -cut. (The strong $\alpha$-cut of the fuzzy real number $X$, for $0 \leq \alpha \leq 1$ is the set $\{t \in R: X(t)>\alpha\})$. 
For $X, Y \in R(I)$ consider a partial ordering $\leq$ as

$$
X \leq Y \text { if and only if } a_{1}^{\alpha} \leq a_{2}^{\alpha} \text { and } b_{1}^{\alpha} \leq b_{2}^{\alpha} \text {, for all } \alpha \in(0,1],
$$

where $[X]^{\alpha}=\left[a_{1}^{\alpha}, b_{1}^{\alpha}\right]$ and $[Y]^{\alpha}=\left[a_{2}^{\alpha}, b_{2}^{\alpha}\right]$.

Let $X, Y \in R(I)$ and $\alpha$-level sets be $[X]^{\alpha}=\left[a_{1}^{\alpha}, b_{1}^{\alpha}\right],[Y]^{\alpha}=\left[a_{2}^{\alpha}, b_{2}^{\alpha}\right]$, $\alpha \in[0,1]$. Then the arithmetic operations on $R(I)$ in terms of $\alpha$-level sets are defined as follows:

$$
\begin{aligned}
& {[X \oplus Y]^{\alpha}=\left[a_{1}^{\alpha}+a_{2}^{\alpha}, b_{1}^{\alpha}+b_{2}^{\alpha}\right],} \\
& {[X \ominus Y]^{\alpha}=\left[a_{1}^{\alpha}-b_{2}^{\alpha}, b_{1}^{\alpha}-a_{2}^{\alpha}\right],} \\
& {[X \otimes Y]^{\alpha}=\left[\min _{i, j \in\{1,2\}} a_{i}^{\alpha} b_{j}^{\alpha}, \max _{i, j \in\{1,2\}} a_{i}^{\alpha} b_{j}^{\alpha}\right]}
\end{aligned}
$$

and $\quad[\overline{1} \div Y]^{\alpha}=\left[\frac{1}{b_{2}^{\alpha}}, \frac{1}{a_{2}^{\alpha}}\right], 0 \notin Y$.

The absolute value, $|X|$ of $X \in R(I)$ is defined by (see for instance Kaleva and Seikkala [4])

$$
|X|(t)= \begin{cases}\max (X(t), X(-t)), & \text { for } t \geq 0 \\ 0, & \text { for } t<0\end{cases}
$$

A fuzzy real number $X$ is called non-negative if $X(t)=0$, for all $t<0$. The set of all non-negative fuzzy real numbers is denoted by $R^{*}(I)$.

\section{Fuzzy Normed Linear Space}

Let $X$ be a linear space over $R$ and the mapping $\|\cdot\|: X \rightarrow R^{*}(I)$ and the mappings $L, M:[0,1] \times[0,1] \rightarrow[0,1]$ be symmetric, non-decreasing in both arguments and satisfy $L(0,0)=0$ and $M(1,1)=1$. Write $[\|x\|]^{\alpha}=\left[\|\| x\left\|\left.\right|_{1} ^{\alpha},\right\|\|x\|_{2}^{\alpha},\right]$, for $x \in X, 0<\alpha \leq 1$ and suppose for all $x \in$ $X, x \neq 0$, there exists $\alpha_{0} \in(0,1]$ independent of $x$ such that for all $\alpha \leq \alpha_{0}$,

(A) $|\|x\||_{2}^{\alpha}<\infty$

(B) inf ${ }_{\alpha \in(0,1]}|||x| \|_{1}^{\alpha}>0$.

The quadruple $(X,\|\cdot\|, L, M)$ is called a fuzzy normed linear space and $\|\cdot\|$ a fuzzy norm on the linear space $X$, if

i) $\|x\|=\overline{0}$ if and only if $x=0$,

ii) $\|r x\|=|r|\|x\|, x \in X, r \in R$, 
iii) for all $x, y \in X$,

(a) $\|x+y\|(s+t) \geq L(\|x\|(s),\|y\|(t))$, whenever $s \leq\|x\|\left\|_{1}^{1}, t \leq\right\| y \|_{1}^{1}$ and $s+t \leq\|x+y\|_{1}^{1}$,

(b) $\|x+y\|(s+t) \geq M(\|x\|(s),\|y\|(t))$, whenever $s \geq\||| x \mid\|_{1}^{1}, t \geq\|y\|_{1}^{1}$ and $s+t \geq\|x+y\|_{1}^{1}$.

In the sequel we take $L(x, y)=\min (x, y)$ and $M(x, y)=\max (x, y)$ for $x, y \in[0,1]$ and we denote $(X,\|\cdot\|, L, M)$ by $(X,\|\cdot\|)$ or simply by $X$ in this case.

With these $L(x, y)=\min (x, y)$ and $M(x, y)=\max (x, y)$ for $x, y \in$ $[0,1]$, we have (refer to Felbin [3]) in a fuzzy normed linear space $(X,\|\cdot\|)$, the triangle inequality (iii) of the definition of fuzzy normed linear space is equivalent to

$$
\|x+y\| \leq\|x\| \oplus\|y\|
$$

The set $\omega(X)$ of all sequences in a vector space $X$ is a vector space with respect to pointwise addition and scalar multiplication. Any subspace $\lambda(X)$ of $\omega(X)$ is called vector valued sequence space. When $(X,\|\cdot\|)$ is a fuzzy normed linear space, then $\lambda(X)$ is called a fuzzy normed linear space-valued sequence space.

Throughout fnls denotes fuzzy normed linear space.

A fnls-valued sequence space $E^{F}(X)$ is said to be normal (or solid) if $\left(y_{k}\right) \in E^{F}(X)$, whenever $\left\|y_{k}\right\| \leq\left\|x_{k}\right\|$, for all $k \in N$ and $\left(x_{k}\right) \in E^{F}(X)$.

Let $K=k_{1}<k_{2}<k_{3} \ldots \subseteq N$ and $E^{F}(X)$ be a fnls-valued sequence space. A $K$-step space of $E^{F}(X)$ is a space of sequences $\lambda_{k}^{E^{F}}(X)=\left\{\left(x_{k_{n}}\right) \in\right.$ $\left.\omega^{F}(X):\left(x_{n}\right) \in E^{F}(X)\right\}$.

A canonical pre-image of a sequence $\left(x_{k_{n}}\right) \in \lambda_{k}^{E^{F}}(X)$ is a sequence $\left(y_{n}\right) \in \omega^{F}(X)$, defined as follows:

$$
y_{n}= \begin{cases}x_{n}, & \text { for } n \in K \\ \overline{0}, & \text { otherwise }\end{cases}
$$

A canonical pre-image of a step space $\lambda_{k}^{E^{F}}(X)$ is a space of canonical pre-images of all elements in $\lambda_{k}^{E^{F}}(X)$, i.e. $y$ is in canonical pre-image $\lambda_{k}^{E^{F}}(X)$ if and only if $y$ is canonical pre-image of some $x \in \lambda_{k}^{E^{F}}(X)$.

A fnls-valued sequence space $E^{F}(X)$ is said to be monotone if $E^{F}(X)$ contains the canonical pre-images of all its step spaces.

From the above definitions we have following remark. 
Remark 2.1. A fnls-valued sequence space $E^{F}(X)$ is solid $\Rightarrow E^{F}(X)$ is monotone.

A fnls-valued sequence space $E^{F}(X)$ is said to be symmetric if $\left(x_{\pi(n)}\right) \in$ $E^{F}(X)$, whenever $\left(x_{k}\right) \in E^{F}(X)$, where $\pi$ is a permutation of $N$.

A fnls-valued sequence space $E^{F}(X)$ is said to be convergence free if $\left(y_{k}\right) \in E^{F}(X)$, whenever $\left(x_{k}\right) \in E^{F}(X)$ and $x_{k}=0$ implies $y_{k}=0$.

Let $(X,\|\cdot\|)$ be a fuzzy normed linear space. A sequence $\left(x_{n}\right) \in X$ is said to converge to $x \in X$, denoted by $\lim _{n \rightarrow \infty} x_{n}=x$, if and only if $\lim _{n \rightarrow \infty}\left\|x_{n}-x\right\|=\overline{0}$.

i.e., $\lim _{n \rightarrow \infty}\left|\left\|x_{n}-x\left|\left\|_{1}^{\alpha}=\lim _{n \rightarrow \infty}\left|\left\|x_{n}-x \mid\right\|_{2}^{\alpha}=0\right.\right.\right.\right.\right.$, for $\alpha \in(0,1]$.

Thus, $\lim _{n \rightarrow \infty}\left\|x_{n}-x\right\|=\overline{0}$ if and only if $\lim _{n \rightarrow \infty}\|\| x_{n}-x \|_{2}^{\alpha}=0$, for $0 \in(0,1]$. if

A sequence $\left(x_{n}\right)$ in a fuzzy normed linear space $(X,\|\cdot\|)$ is called Cauchy

$\lim _{\substack{n \rightarrow \infty \\ m \rightarrow \infty}}\left\|x_{m}-x_{n}\right\|=\overline{0}$

i.e., $\lim _{\substack{n \rightarrow \infty \\ m \rightarrow \infty}}\left|\left\|x_{n}-x \mid\right\|_{2}^{\alpha}=0\right.$, for $\alpha \in(0,1]$.

A fuzzy normed linear space $(X,\|\cdot\|)$ is called fuzzy complete if every Cauchy sequence in $X$ converges in $X$.

With the concept of fuzzy norm $\|\cdot\|$, the fuzzy normed linear space valued sequence space $\ell_{p}^{F}(X)$ is defined by:

$$
\ell_{p}^{F}(X)=\left\{x=\left(x_{k}\right) \in \omega^{F}(X): \sum_{k=1}^{\infty}\left\|x_{k}\right\|^{p} \leq \lambda, \text { for some } \lambda \in R^{*}(I)\right\}
$$

Let $(X,\|\cdot\|)$ be a fuzzy normed linear space. Then for a sequence $x=\left(x_{k}\right) \in \ell_{p}^{F}(X), 1 \leq p<\infty$, the class of all $p$-absolutely summable sequences in $(X,\|\cdot\|)$ we define

$$
\|x\|=\left\{\sum_{k=1}^{\infty}\left\|x_{k}\right\|^{p}\right\}^{\frac{1}{p}}
$$

Clearly, $\|x\|$ is a norm. 
Throughout $\omega^{F}(X), \ell_{p}^{F}(X)$ and $c^{F}(X)$ denote the spaces of all, p-absolutely summable and convergent sequences in fuzzy normed linear space $X$ respectively.

\section{Main results}

Theorem 3.1. The class of p-absolutely summable sequences, $\ell_{p}^{F}(X)$ is fnls-valued sequence space.

Proof: Let $(X,\|\cdot\|)$ be a fnls and $x=\left(x_{k}\right), y=\left(y_{k}\right) \in \ell_{p}^{F}(X)$. We have for $k \in N,\left\|x_{k}+y_{k}\right\|^{p} \leq 2^{p} \max \left\{\left\|x_{k}\right\|^{p},\left\|y_{k}\right\|^{p}\right\} \leq 2^{p}\left(\left\|x_{k}\right\|^{p} \oplus\left\|y_{k}\right\|^{p}\right)$.

It follows that $\sum_{k=1}^{\infty}\left\|x_{k}+y_{k}\right\|^{p}<\infty$. Thus $\left(x_{k}+y_{k}\right) \in \ell_{p}^{F}(X)$.

Let $r \in R$. We have $\sum_{k=1}^{\infty}\left\|r x_{k}\right\|^{p}=|r|^{p} \sum_{k=1}^{\infty}\left\|x_{k}\right\|^{p}<\infty$. Thus $r x_{k} \in \ell_{p}^{F}(X)$.

So, $\ell_{p}^{F}(X)$ is a subspace of $\omega^{F}(X)$ and hence it is fnls-valued sequence space.

Theorem 3.2. The space $\ell_{p}^{F}(X), 1 \leq p<\infty$, is complete with the norm

$$
\|x\|=\left\{\sum_{k=1}^{\infty}\left\|x_{k}\right\|^{p}\right\}^{\frac{1}{p}},
$$

where $x=\left(x_{k}\right) \in \ell_{p}^{F}(X), k \in N$ and $X$ is complete.

Proof: Let $\left(x^{(n)}\right)$ be a Cauchy sequence in $\ell_{p}^{F}(X)$,

where $x^{(n)}=\left(x_{k}^{(n)}\right)=\left(x_{1}^{(n)}, x_{2}^{(n)}, x_{3}^{(n)}, \ldots\right) \in \ell_{p}^{F}$, for all $n \in N$.

Then for a given $\bar{\varepsilon}>0$ there exists $n_{0} \in N$ such that $\left\|x^{(n)}-x^{(m)}\right\|=$ $\left\{\sum\left\|x_{k}^{(n)}-x_{k}^{(m)}\right\|^{p}\right\}^{\frac{1}{p}}<\bar{\varepsilon}$, for all $m, n \geq n_{0}$.

$\Rightarrow\left\|x_{k}^{(n)}-x_{k}^{(m)}\right\|<\bar{\varepsilon}$.

$\Rightarrow\left(x_{k}^{(n)}\right)$ is a Cauchy sequence in $X$ for each $k \in N$.

Since $X$ complete, there exists $x_{k} \in X$ such that $\left\|x_{k}^{(n)}-x_{k}\right\| \rightarrow \overline{0}$ as $n \rightarrow \infty$, for each $k$.

$\Rightarrow\|\| x_{k}^{(n)}-x_{k} \|_{2}^{\alpha} \rightarrow 0$ as $n \rightarrow \infty$, for each $\alpha \in(0,1]$.

Again, $x^{(n)}$ is a Cauchy sequence, for each $\bar{\varepsilon}>0$, there exists $n_{0}=n_{0}(\varepsilon)$ such that

$$
\left\{\sum\left\|x_{k}^{(n)}-x_{k}^{(m)}\right\|^{p}\right\}^{\frac{1}{p}}<\bar{\varepsilon}
$$


$\Rightarrow\left[\sum\left\{\left\|\mid x_{k}^{(n)}-x_{k}^{(m)}\right\|_{2}^{\alpha}\right\}^{p}\right]^{\frac{1}{p}}<\varepsilon$ for each $\alpha \in(0,1]$.

Now fix $n \geq n_{0}$ and let $m \rightarrow \infty$, we have $\left[\sum\left\{\left\|\mid x_{k}^{(n)}-x_{k}\right\|_{2}^{\alpha}\right\}^{p}\right]^{\frac{1}{p}} \leq \varepsilon$, for all $n \geq n_{0}$ and $\alpha \in(0,1]$

$$
\Rightarrow\left\{\sum\left\|x_{k}^{(n)}-x_{k}\right\|^{p}\right\}^{\frac{1}{p}} \leq \bar{\varepsilon}, \text { for all } n \geq n_{0} .
$$

$$
\Rightarrow\left\{\sum\left\|x^{(n)}-x\right\|^{p}\right\}^{\frac{1}{p}} \leq \bar{\varepsilon}, \text { for all } n \geq n_{0}, \text { where } x=\left(x_{k}\right)
$$

Hence $x^{(n)}$ as $n \rightarrow \infty$.

Now we show that $x=\left(x_{k}\right) \in \ell_{p}^{F}(X)$.

From (3.2) we have, $\left[\left\|x^{(n)}-x\right\|^{p}\right] \leq \bar{\varepsilon}^{p}$

$\Rightarrow\left(x^{(n)}-x\right) \in \ell_{p}^{F}(X)$.

Since $x=x^{(n)}+\left(x-x^{(n)}\right)$, so by Minkowski inequality and using (3.1) we have

$$
\begin{aligned}
& \left\{\sum_{k=1}^{\infty}\left\|x_{k}\right\|^{p}\right\}^{\frac{1}{p}} \leq\left\{\sum_{k=1}^{\infty}\left\|x_{k}^{(n)}\right\|^{p}\right\}^{\frac{1}{p}} \oplus\left\{\sum_{k=1}^{\infty}\left\|x_{k}-x_{k}^{(n)}\right\|^{p}\right\}^{\frac{1}{p}} \\
& \leq\left\{\sum_{k=1}^{\infty}\left\|x_{k}^{(n)}\right\|^{p \frac{1}{p}} \oplus \bar{\varepsilon}\right. \\
& \Rightarrow\left\{\sum_{k=1}^{\infty}\left\|x_{k}\right\|^{p}\right\}<\infty, \text { since } x^{(n)}=\left(x_{k}^{(n)}\right) \in \ell_{p}^{F}(X) .
\end{aligned}
$$

Thus $x \in \ell_{p}^{F}(X)$ and hence the result.

Theorem 3.3. The sequence space $\ell_{p}^{F}(X)$ is solid and as such is monotone.

Proof: Consider two sequences $x=\left(x_{k}\right)$ and $y=\left(y_{k}\right)$ such that $\left\|x_{k}\right\| \leq$ $\left\|y_{k}\right\|$, for all $k \in N$ and $\left(y_{k}\right) \in \ell_{p}^{F}(X)$.

$\Rightarrow \sum\left\|x_{k}\right\|^{p} \leq \sum\left\|y_{k}\right\|^{p}<\infty$. Thus $x=\left(x_{k}\right) \in \ell_{p}^{F}(X)$.

Hence $\ell_{p}^{F}(X)$ is solid.

The space $\ell_{p}^{F}(X)$ is monotone follows from the remark 2.1 .

Theorem 3.4. The sequence space $\ell_{p}^{F}(X)$ is not convergence free.

Proof: The result follows from the following example. 
Example 3.1. Consider the sequence $x=\left(x_{k}\right)$ defined as follows.

$$
x_{k}= \begin{cases}k^{-2}, & \text { for } k \text { even } \\ 0, & \text { for } k \text { odd }\end{cases}
$$

We have $X$ is a fuzzy normed linear space. For any sequence $z=\left(z_{k}\right) \in X$, let us consider $\left\|z_{k}\right\|$, defined as follows:

For $k \in N, z_{k} \neq 0,\left\|z_{k}\right\|(t)= \begin{cases}\frac{4 t}{z_{k} \mid}-3, & \text { for } \frac{3\left|z_{k}\right|}{4} \leq t \leq\left|z_{k}\right|, \\ 0, & \text { otherwise. }\end{cases}$

and for $z_{k}=0,\left\|z_{k}\right\|(t)= \begin{cases}1, & \text { for } t=0 \\ 0, & \text { otherwise. }\end{cases}$

Using (3), we have for

$k$ even and $x_{k} \neq 0,\left\|x_{k}\right\|(t)= \begin{cases}\frac{4 t}{\left|x_{k}\right|}-3, & \text { for } \frac{3\left|x_{k}\right|}{4} \leq t \leq\left|x_{k}\right|=k^{-2}, \\ 0, & \text { otherwise. }\end{cases}$

and for $k$ odd,

$$
\left\|x_{k}\right\|(t)= \begin{cases}1, & \text { for } t=0 \\ 0, & \text { otherwise }\end{cases}
$$

Again for each $\alpha \in(0,1]$ we have,

$$
\left[\left\|x_{k}\right\|\right]^{\alpha}= \begin{cases}{\left[\frac{(\alpha+3)}{4} k^{-2}, k^{-2}\right],} & \text { for } k \text { even, } \\ {[0,0],} & \text { for } k \text { odd }\end{cases}
$$

Hence for each $\alpha \in(0,1], \sum_{k=1}^{\infty}\left[\left\||| x_{k} \mid\right\|_{2}^{\alpha}\right]^{p}=\sum_{k=1}^{\infty}\left(\frac{1}{k^{2}}\right)^{p}-\sum_{k=0}^{\infty}\left\{\frac{1}{(2 k+1)^{2}}\right\}^{p}<\infty$ $\Rightarrow \sum_{k=1}^{\infty}\left\|x_{k}\right\|^{p}<\infty$. Thus $x=\left(x_{k}\right) \in \ell_{p}^{F}(X)$.

Let the sequence $y=\left(y_{k}\right)$ be defined as follows:

$$
y_{k}= \begin{cases}k^{-\frac{1}{p}}, & \text { for } k \text { even } \\ 0, & \text { for } k \text { odd }\end{cases}
$$

Then for $k$ even and using (3.1), we have 


$$
\left\|y_{k}\right\|(t)= \begin{cases}\frac{4 t}{\left|y_{k}\right|}-3, & \text { for } \frac{3\left|y_{k}\right|}{4} \leq t \leq\left|y_{k}\right|=k^{-\frac{1}{p}}, \\ 0, & \text { otherwise. }\end{cases}
$$

and for $k$ odd, $\left\|y_{k}\right\|(t)= \begin{cases}1, & \text { for } t=0 \\ 0, & \text { otherwise }\end{cases}$

Again for each $\alpha \in(0,1]$ we have,

$$
\left[\left\|y_{k}\right\|\right]^{\alpha}= \begin{cases}{\left[\frac{(\alpha+3)}{4} k^{-\frac{1}{p}}, k^{-\frac{1}{p}}\right],} & \text { for } k \text { even } \\ {[0,0],} & \text { for } k \text { odd }\end{cases}
$$

Hence for each $\alpha \in(0,1], \sum_{k=1}^{\infty}\left[||\left|y_{k}\right| \|_{2}^{\alpha}\right]^{p}=\sum_{k=1}^{\infty}\left(k^{-\frac{1}{p}}\right)^{p}-\sum_{k=0}^{\infty}\left\{(2 k+1)^{-\frac{1}{p}}\right\}^{p}$, which is unbounded.

$$
\Rightarrow \sum_{k=1}^{\infty}\left\|y_{k}\right\|^{p} \text { is unbounded. Thus } y=\left(y_{k}\right) \notin \ell_{p}^{F}(X) \text {. }
$$

Hence $\ell_{p}^{F}(X)$ is not convergence free.

Theorem 3.5. The sequence space $\ell_{p}^{F}(X)$ is symmetric.

Proof: Let $x=\left(x_{k}\right) \in \ell_{p}^{F}(X)$.

Let $y=\left(y_{k}\right)$ be a rearrangement of the sequence $x=\left(x_{k}\right)$ such that $x_{k}=y_{m_{k}}$ for each $k \in N$.

Then, $\sum\left\|y_{m_{k}}\right\|^{p}=\sum\left\|x_{k}\right\|^{p}<\infty$.

Thus $y=\left(y_{k}\right) \in \ell_{p}^{F}(X)$. Hence the result.

Theorem 3.6. $\ell_{p}^{F}(X) \subseteq \ell_{q}^{F}(X), 1 \leq p \leq q<\infty$.

Proof: Let $x=\left(x_{k}\right) \in \ell_{p}^{F}(X)$, then we have

$\sum\left\|x_{k}\right\|^{p}<\infty \Rightarrow \sum\left[\|\| x_{k} \mid \|_{2}^{\alpha}\right]^{p}<\infty$, for each $\alpha \in(0,1]$.

Since $\lim _{k \rightarrow \infty}\left\|x_{k}-0\right\|=\overline{0}\left(\right.$ as $\left.\left(x_{k}\right) \in \ell_{p}^{F}(X)\right)$, so there exists a positive integer $n_{0}$ such that $\left\|x_{k}-0\right\| \leq \overline{1}\left(\overline{1} \in R^{*}(I)\right)$, for all $k \geq n_{0}$.

We have, $\sum\left\|x_{k}\right\|^{q}=\sum_{k=1}^{n_{0}-1}\left\|x_{k}\right\|^{q} \oplus \sum_{k=n_{0}}^{\infty}\left\|x_{k}\right\|^{q}$.

Clearly, $\sum_{k=n_{0}}^{\infty}\left\|x_{k}\right\|^{q} \leq \sum_{k=n_{0}}^{\infty}\left\|x_{k}\right\|^{p}<\infty$.

Further we have, $\sum_{k=1}^{n_{0}-1}\left\|x_{k}\right\|^{q}$ is finite sum. So, $\sum\left\|x_{k}\right\|^{q}<\infty$.

Thus $x=\left(x_{k}\right) \in \ell_{q}^{F}(X)$ and hence the result. 


\section{References}

[1] Das, P. C. : Statistically convergent fuzzy sequence spaces by fuzzy metric; Kyungpook Math. Journal, 54 (3), pp. 413-423, (2014).

[2] Das, P. C. : p-absolutely Summable Type Fuzzy Sequence Spaces by Fuzzy Metric; Boletim da Sociedade Paranaense de Matemtica, 32 (2), pp. 35-43, (2014).

[3] Felbin, C. : Finite dimensional fuzzy normed linear space; Fuzzy Sets and Systems, 48, pp. 239-248, (1992).

[4] Kelava, O. and Seikkala, S. : On fuzzy metric spaces; Fuzzy Sets and Systems, 12, pp. 215-229, (1984).

[5] Matloka, M. : Sequences of fuzzy numbers ; BUSEFAL, 28, pp. 28-37, (1986).

[6] Subrahmanyam, P. V. : Cesaro Summability for fuzzy real numbers; J. Analysis, 7, pp. 159-168, (1999).

[7] Tripathy, B. C. Baruah, A. Et, M. and Gungor, M. : On almost statistical convergence of new type of generalized difference sequence of fuzzy numbers; Iranian Journal of Science and Technology, Transacations A Science, 36 (2), pp. 147-155, (2012).

[8] Tripathy, B. C. and Dutta, A. J. : Statistically pre-Cauchy fuzzy realvalued sequences defined by Orlicz function; Proyecciones Journal of Mathematics, 33 (3), pp. 235-243, (2014).

[9] Tripathy, B. C. and Dutta, A. J. : Lacunary I-convergent sequences of fuzzy real numbers; Proyecciones Journal of Mathematics, 34 (3), pp. 205-218, (2015).

[10] Tripathy, B. C. and Sarma, B. : Sequence spaces of fuzzy real numbers defined by Orlicz functions; Math. Slovaca, 58 (5), pp. 621-628, (2008).

[11] Tripathy, B. C. and Debnath, S. : On generalized difference sequence spaces of fuzzy numbers; Acta Scientiarum Technology, 35 (1), pp. 117-121, (2013). 


\section{Paritosh Chandra Das}

Department of Mathematics,

Rangia College,

Rangia-781354; Assam,

INDIA

e-mail : pcdasrc2011@gmail.com 